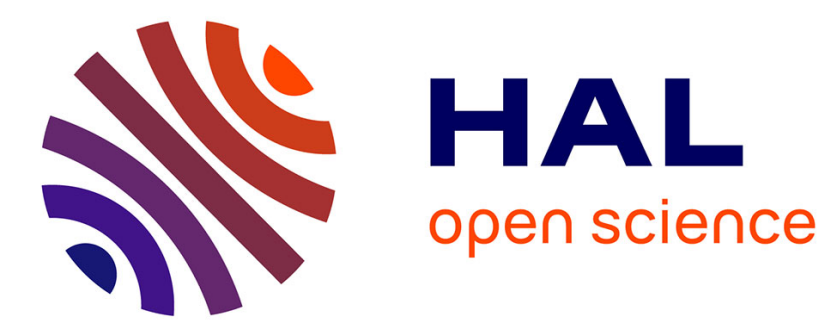

\title{
Nombre maximal d'hyperplans instables pour un fibré de Steiner
}

Jean Vallès

\section{To cite this version:}

Jean Vallès. Nombre maximal d'hyperplans instables pour un fibré de Steiner. Mathematische Zeitschrift, 2000. hal-01991069

\section{HAL Id: hal-01991069 \\ https://hal.science/hal-01991069}

Submitted on 23 Jan 2019

HAL is a multi-disciplinary open access archive for the deposit and dissemination of scientific research documents, whether they are published or not. The documents may come from teaching and research institutions in France or abroad, or from public or private research centers.
L'archive ouverte pluridisciplinaire HAL, est destinée au dépôt et à la diffusion de documents scientifiques de niveau recherche, publiés ou non, émanant des établissements d'enseignement et de recherche français ou étrangers, des laboratoires publics ou privés. 


\title{
Nombre maximal d'hyperplans instables pour un fibré de Steiner
}

\author{
Jean Vallès
}

Algebraic Geometry

RÉSUMÉ - Soit $\mathcal{S}_{n, k}$ la famille des fibrés de Steiner $S$ sur $\mathbf{P}_{n}$ définis par une suite exacte $(k>0)$

$$
0 \rightarrow k O_{\mathbf{P}_{n}}(-1) \longrightarrow(n+k) O_{\mathbf{P}_{n}} \longrightarrow S \rightarrow 0
$$

Nous montrons le résultat suivant : Soient $S \in \mathcal{S}_{n, k}$ et $H_{1}, \cdots, H_{n+k+2}$ des hyperplans distincts tels que $h^{0}\left(S_{H_{i}}^{\vee}\right) \neq 0$. Alors il existe une courbe rationnelle normale $C_{n} \subset \mathbf{P}_{n}^{\vee}$ telle que $H_{i} \in C_{n}$ pour $i=1, \ldots, n+k+2$ et $S \simeq E_{n+k-1}\left(C_{n}\right)$, où $E_{n+k-1}\left(C_{n}\right)$ est le fibré de Schwarzenberger sur $\mathbf{P}_{n}$ appartenant à $\mathcal{S}_{n, k}$ associé à la courbe $C_{n} \subset \mathbf{P}_{n}^{\vee}$.

On en déduit qu'un fibré de Steiner $S \in \mathcal{S}_{n, k}$, s'il n'est pas un fibré de Schwarzenberger, possède au plus $(n+k+1)$ hyperplans instables; ceci prouve dans tous les cas un résultat de Dolgachev et Kapranov ([DK], thm. 7.2) concernant les fibrés logarithmiques.

ABSTRACT - Let $\mathcal{S}_{n, k}$ denote the family of Steiner's bundle $S$ on $\mathbf{P}_{n}$ defined by the exact sequence $(k>0)$

$$
0 \rightarrow k O_{\mathbf{P}_{n}}(-1) \longrightarrow(n+k) O_{\mathbf{P}_{n}} \longrightarrow S \rightarrow 0
$$

We show the following result : Let $S \in \mathcal{S}_{n, k}$ and $H_{1}, \cdots, H_{n+k+2}$ distincts hyperplanes such that $h^{0}\left(S_{H_{i}}^{\vee}\right) \neq 0$. Then it exists a rational normal curve $C_{n} \subset \mathbf{P}_{n}^{\vee}$ such that $H_{i} \in C_{n}$ for $i=1, \ldots, n+k+2$ and $S \simeq E_{n+k-1}\left(C_{n}\right)$, where $E_{n+k-1}\left(C_{n}\right)$ is the Schwarzenberger's bundle on $\mathbf{P}_{n}$ which belongs to $\mathcal{S}_{n, k}$ associated to $C_{n} \subset \mathbf{P}_{n}^{\vee}$

It implies that a Steiner's bundle $S \in \mathcal{S}_{n, k}$, if it isn't a Schwarzenberger's bundle, possesses no more than $(n+k+1)$ unstable hyperplanes; this proves in any case a result of Dolgachev and Kapranov ([DK], thm 7.2) about logarithmic bundles.

\section{Introduction}

Soient $\mathcal{H}=\left\{H_{1}, \cdots, H_{n+k+1}\right\}$ un arrangement de $(n+k+1)$-hyperplans de $\mathbf{P}_{n}=\mathbf{P}_{n}(\mathbf{C})$ (on dira aussi par abus de langage qu'un hyperplan $H$ de $\mathbf{P}_{n}$ est un point de $\mathbf{P}_{n}^{\vee}$ ) en position linéaire générale et $E(\mathcal{H})=\Omega_{\mathbf{P}_{n}}\left(\log \bigcup H_{i}\right)$ le fibré logarithmique associé à $\mathcal{H}$ (i.e. le fibré vectoriel des 1-formes différentielles sur $\mathbf{P}_{n}$ à pôles logarithmiques sur le diviseur $\bigcup H_{i}$, voir [De]). Dans [DK] les auteurs montrent que $E(\mathcal{H}) \in \mathcal{S}_{n, k}$ (théorème 3.5 ) où $\mathcal{S}_{n, k}$ est la famille des fibrés de Steiner $S$ définis par une suite exacte

$$
0 \rightarrow k O_{\mathbf{P}_{n}}(-1) \longrightarrow(n+k) O_{\mathbf{P}_{n}} \longrightarrow S \rightarrow 0
$$

Dolgachev et Kapranov conjecturent que pour $k>0$ l'association $\mathcal{H} \rightarrow E(\mathcal{H})$ est bijective sauf si $\mathcal{H} \in C_{n}$ où $C_{n} \in \mathbf{P}_{n}^{\vee}$ est une courbe rationnelle normale; dans ce cas le fibré logarithmique est le fibré de Schwarzenberger $E_{n+k-1}\left(C_{n}\right)$ ([Sch1]) associé à $C_{n}$. Ils montrent 
ce résultat pour $k \geq n+2$, en étudiant les droites de saut de $E(\mathcal{H})$ (théorème 7.2 , dit de Torelli).

Dans cet article nous prouvons ce résultat pour tout $k>0$ (corollaire 3.1). Notre preuve repose sur l'étude des restrictions de $E(\mathcal{H})$, non pas aux droites, mais aux hyperplans de $\mathbf{P}_{n}$. En effet il résulte de [DK] prop.2.3 que $h^{0}\left(E(\mathcal{H})_{H_{i}}^{\vee}\right) \neq 0$ pour $i=1, \cdots, n+k+1$. Plus généralemment on définit dans $\mathbf{P}_{n}^{\vee}$ l'ensemble $W(S)$ des hyperplans instables d'un fibré de Steiner $S \in \mathcal{S}_{n, k}$ de la manière suivante

$$
H \in W(S) \Leftrightarrow h^{0}\left(S_{H}^{\vee}\right) \neq 0
$$

Le résultat de Dolgachev et Kapranov est une conséquence directe du théorème suivant qui affirme qu'un fibré de Steiner $S \in \mathcal{S}_{n, k}$, s'il n'est pas un fibré de Schwarzenberger, possède au plus $(n+k+1)$ hyperplans instables.

Théorème 3.1. Soient $S \in \mathcal{S}_{n, k}$ et $H_{1}, \cdots, H_{n+k+2}$ des hyperplans distincts tels que $h^{0}\left(S_{H_{i}}^{\vee}\right) \neq 0$. Alors il existe une courbe rationnelle normale $C_{n} \subset \mathbf{P}_{n}^{\vee}$ telle que $H_{i} \in C_{n}$ pour $i=1, \ldots, n+k+2$ et $S \simeq E_{n+k-1}\left(C_{n}\right)$.

On vérifie par ailleurs que $W\left(E_{n+k-1}\left(C_{n}\right)\right)=C_{n}$ (proposition 2.2).

Dans la première partie on montre (proposition 2.1) que si $S \in \mathcal{S}_{n, k}$ avec $k>1$ et $H_{1} \in W(S)$ alors l'homomorphisme $S \rightarrow O_{H_{1}}$ induit par une section non nulle de $S_{H_{1}}^{\vee}$ est surjectif et son noyau $T_{1}$ vérifie $T_{1} \in \mathcal{S}_{n, k-1}$ et $W(S) \subset W\left(T_{1}\right) \cup H$.

Après $k-2$ réductions on se ramène ainsi à un fibré de Steiner $T_{k-2} \in \mathcal{S}_{n, 2}$. Comme tout fibré de Steiner de $\mathcal{S}_{n, 2}$ est un fibré de Schwarzenberger associé à une courbe rationnelle normale $C_{n} \subset \mathbf{P}_{n}^{\vee}$ ([DK], prop 6.8) on en déduit que $T_{k-2} \simeq E_{n+1}\left(C_{n}\right)$ et que les hyperplans instables de $S$ sont des points de $C_{n}$.

On conclut en utilisant la proposition 2.3 établie dans la deuxième partie qui affirme qu'un fibré de Steiner $S \in \mathcal{S}_{n, k}$ qui possède $n+k+1$ hyperplans instables osculateurs d'une même courbe rationnelle normale est un fibré de Schwarzenberger (ce résultat généralise le théorème 6.4 de [DK] concernant les fibrés logarithmiques).

Dans la suite de ce texte nous écrirons c.r.n. pour courbe rationnelle normale.

Ce problème d'existence d'un nombre maximal d'hyperplans instables pour un fibré de Steiner m'a été posé par V.Ancona et G.Ottaviani. Je tiens à les remercier très chaleureusement pour cela ainsi que pour les nombreuses discussions que nous avons eues sur ce thème.

Ce travail a été réalisé dans le cadre d'un contrat de recherche européen (programme capital humain et mobilité) entre La Scuola Normale Superiore di Pisa et L'Université P.et M. Curie Paris VI.

\section{Les fibrés de Steiner}

Définition 2.1. Un fibré vectoriel $S$ de rang $n$ sur $\mathbf{P}_{n}$ est appelé fibré de Steiner s'il existe une suite exacte $(k>0)$

$$
0 \rightarrow k O_{\mathbf{P}_{n}}(-1) \longrightarrow(n+k) O_{\mathbf{P}_{n}} \longrightarrow S \rightarrow 0
$$

Notation: On note $\mathcal{S}_{n, k}$ la famille des fibrés de Steiner définis comme ci-dessus.

Remarque. Pour $k>0$ on a $h^{0}\left(S^{\vee}\right)=0$. Pour $k=1$, le fibré $S$ est isomorphe à $\Omega_{\mathbf{P}_{n}}^{\vee}(-1)$. 
Rappelons qu'un fibré vectoriel $E$ est stable si pour tout sous-faisceau cohérent sans torsion $F \subset E$ on a

$$
\frac{\operatorname{deg}(F)}{\operatorname{rg}(F)}<\frac{\operatorname{deg}(E)}{\operatorname{rg}(E)}
$$

et que les fibrés de Steiner $S$ ainsi définis sont stables ([BS], théorème 2.7).

On dira qu'un hyperplan est instable pour le fibré $S$ s'il appartient à l'ensemble suivant

$$
W(S)=\left\{H \in \mathbf{P}_{n}^{\vee} / h^{0}\left(S_{H}^{\vee}\right) \neq 0\right\}
$$

Proposition 2.1. Soient $S \in \mathcal{S}_{n, k}$ et $H \in W(S)$. Les propriétés suivantes sont vérifiées. 1) $S_{H}=O_{H} \oplus T$ où $T$ est un fibré de Steiner sur $H$ (ou $T=0$ si $n=1$ ).

2) L'homorphisme $S \rightarrow O_{H}$ (induit par une section non nulle de $S_{H}^{\vee}$ ) est surjectif et, pour $k>1$, son noyau, noté $S^{\prime}$, vérifie $S^{\prime} \in \mathcal{S}_{n, k-1}$ et $W(S) \subset W\left(S^{\prime}\right) \cup\{H\}$.

Remarque. Il résulte de 1) que $h^{0}\left(S_{H}^{\vee}\right)=h^{0}\left(O_{H}\right)=1$ et que cette section est partout non nulle.

Preuve proposition 2.1. 1) Une section non nulle de $t \in H^{0}\left(S_{H}^{\vee}\right)$ induit un homomorphisme non nul $t^{\vee}: S_{H} \longrightarrow O_{H}$; notons $T$ son noyau. L'homorphisme composé

$$
H^{0}(S) \otimes O_{H} \rightarrow S_{H} \stackrel{t^{\vee}}{\rightarrow} O_{H}
$$

est non nul donc il est surjectif. Ceci prouve que $t^{\vee}$ est surjectif et que l'application $H^{0}\left(S_{H}\right) \rightarrow H^{0}\left(O_{H}\right)$ est surjective. On en déduit que $T$ est un fibré vectoriel de rang $n-1$ sur $H$ et que $S_{H}=O_{H} \oplus T$.

Le lemme du serpent appliqué au diagramme commutatif suivant prouve que $T$ est un fibré de Steiner sur $H$.

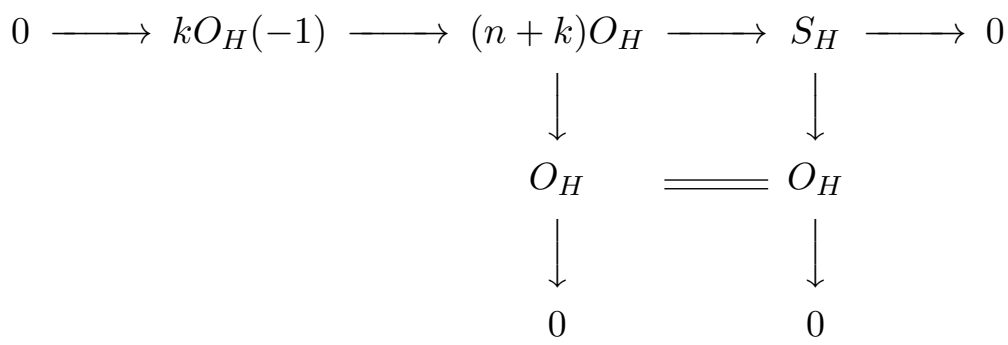

2) Il résulte de 1) que l'homorphisme composé $S \rightarrow S_{H} \rightarrow O_{H}$ induit par la section $t \in H^{0}\left(S_{H}^{\vee}\right)$ est surjectif. Son noyau (noté $S^{\prime}$ ) est un fibré de rang $n$ sur $\mathbf{P}_{n}$. Le lemme du 
serpent appliqué au diagramme commutatif suivant prouve que $S^{\prime}$ est un fibré de Steiner

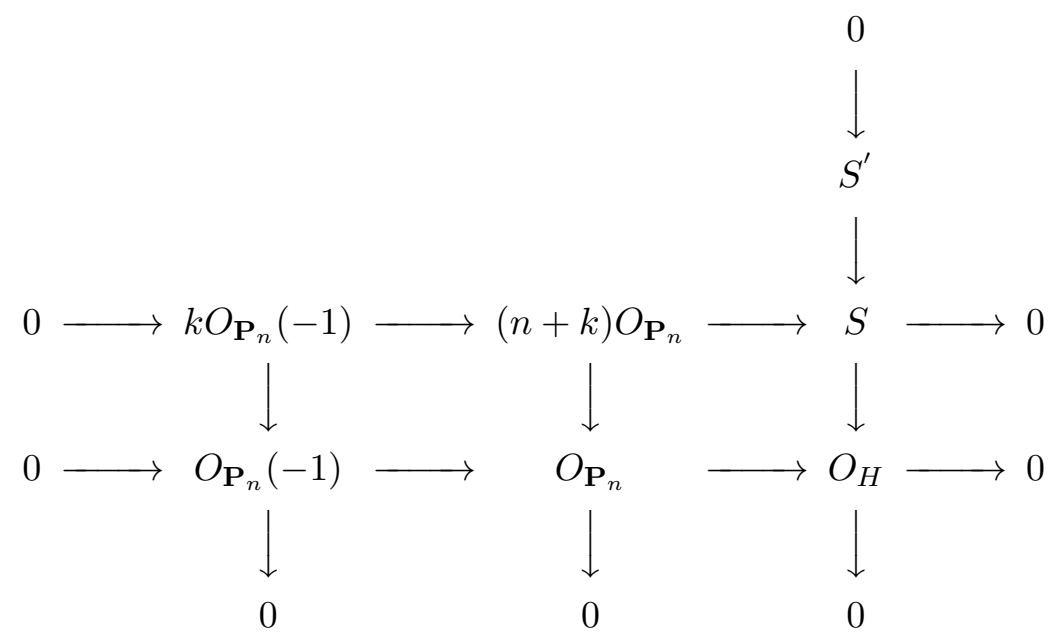

La suite exacte (duale de la dernière colonne du diagramme ci-dessus)

$$
0 \longrightarrow S^{\vee} \longrightarrow S^{\vee} \longrightarrow O_{H}(1) \longrightarrow 0
$$

montre que $W(S) \subset W\left(S^{\prime}\right) \cup\{H\}$.

\subsection{Les fibrés de Schwarzenberger}

Soient $\mathbf{F}$ la variété d'incidence point-hyperplan de $\mathbf{P}_{n}, p$ et $q$ les morphismes de projections $\operatorname{sur} \mathbf{P}_{n}$ et $\mathbf{P}_{n}^{\vee}$.

Considérons le diagramme d'incidence suivant (où $\bar{p}$ et $\bar{q}$ sont les morphismes de projections $p$ et $q$ restreints à $X=q^{-1}\left(C_{n}\right)$ )

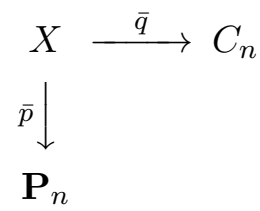

On note $O_{C_{n}}\left(\frac{m}{n}\right)$ le fibré en droite sur $C_{n}$ de degré $m$ correspondant à $O_{\mathbf{P}_{1}}(m)$ via l'isomorphisme $\mathbf{P}_{1} \simeq C_{n}$.

Définition 2.2. Soit $m$ un entier relatif, on appelle fibré de Schwarzenberger associé à $C_{n}$ le fibré vectoriel de rang $n$ suivant

$$
E_{m}\left(C_{n}\right)=\bar{p}_{*} \bar{q}^{*} O_{C_{n}}\left(\frac{m}{n}\right)
$$

Cette définition est donnée par Schwarzenberger ([Sch1], théorème 4). Pour $m \geq n$ les fibrés $E_{m}\left(C_{n}\right)$ sont des fibrés de Steiner ([DK], prop. 6.3 ou [Sch1], prop.2).

Pour $n=2$ le résultat qui suit est démontré par Schwarzenberger ([Sch1], prop.8). Cependant comme sa preuve ne semble pas se généraliser facilement aux cas $n>2$, nous en donnons brièvement une démonstration. 
Proposition 2.2. Lorsque $m>n$ on a $W\left(E_{m}\left(C_{n}\right)\right)=C_{n}$.

Preuve de la proposition 2.2. Notons $\left(X_{0}, \cdots, X_{n}\right)$ les coordonnées homogènes de $\mathbf{P}_{n}$ et considérons la résolution suivante de $E_{m}\left(C_{n}\right)$

$$
0 \rightarrow(m-n+1) O_{\mathbf{P}_{n}}(-1) \stackrel{M}{\longrightarrow}(m+1) O_{\mathbf{P}_{n}} \longrightarrow E_{m}\left(C_{n}\right) \rightarrow 0
$$

où un représentant de la matrice $M$ s'écrit sous la forme connue ([Sch1], prop.2)

$$
M=\left(\begin{array}{ccccc}
0 & 0 & \cdots & 0 & X_{0} \\
\cdots & \cdots & \cdots & X_{0} & X_{1} \\
\cdots & 0 & \cdots & X_{1} & X_{2} \\
0 & X_{0} & \cdots & X_{2} & . \\
X_{0} & X_{1} & \cdots & \cdots & X_{n} \\
X_{1} & X_{2} & \cdots & X_{n} & 0 \\
X_{2} & \cdots & \cdots & 0 & \cdots \\
\cdots & X_{n} & \cdots & \cdots & \cdots \\
X_{n} & 0 & \cdots & 0 & 0
\end{array}\right)
$$

Il est clair qu'un hyperplan $H$ est instable pour $E_{m}\left(C_{n}\right)$ si et seulement s'il existe un vecteur non nul $\left(x_{0}, \cdots, x_{m}\right) \in \mathbf{C}^{m+1}$ tel que les formes linéaires $\left(\sum_{i=0}^{n} x_{i+j} X_{i}\right)_{j=0, \cdots, m-n}$ soient proportionnelles à $H$. On vérifie alors sans difficulté qu'il existe $\left(t_{0}, t_{1}\right) \in \mathbf{C}$ tel que $\left(x_{0}, \cdots, x_{m}\right)=\left(t_{0}^{m}, \cdots, t_{0}^{m-i} t_{1}^{i}, \cdots, t_{1}^{m}\right)$. Ceci prouve, après factorisation de la $j$-ième équation par $t_{0}^{m-n-j} t_{1}^{j}$, que l'équation de $H$ est

$$
\sum_{i=0}^{n} t_{0}^{n-i} t_{1}^{i} X_{i}=0 .
$$

Réciproquement on montre qu'un fibré de Steiner $S \in \mathcal{S}_{n, k}$ qui possède $n+k+1$ hyperplans instables osculateurs d'une c.r.n. est un fibré de Schwarzenberger.

Proposition 2.3. Soient $S \in \mathcal{S}_{n, k}$ et $H_{1}, \cdots, H_{n+k+1}$ des hyperplans instables pour $S$. Alors,

$$
H_{1}, \cdots, H_{n+k+1} \in C_{n} \Rightarrow S \simeq E_{n+k-1}\left(C_{n}\right)
$$

Preuve de la proposition 2.3. Considérons le diagramme d'incidence déjà introduit précédemment

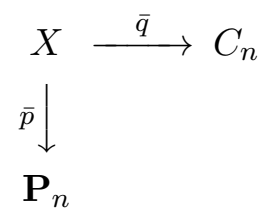

Appliquons le foncteur $\bar{q}_{*} \bar{p}^{*}$ à la suite exacte

$$
0 \rightarrow S^{\vee} \longrightarrow(n+k) O_{\mathbf{P}_{n}} \longrightarrow k O_{\mathbf{P}_{n}}(1) \rightarrow 0
$$

Comme $\bar{q}_{*} \bar{p}^{*} O_{\mathbf{P}_{n}}(1)=\Omega_{\mathbf{P}_{n}^{\vee}}(-1)_{\mid C_{n}}=n O_{C_{n}}\left(\frac{1}{n}\right)$, on obtient un homomorphisme

$$
(n+k) O_{C_{n}} \stackrel{M}{\longrightarrow} n k O_{C_{n}}\left(\frac{1}{n}\right)
$$


Par hypothèse les mineurs maximaux de $M$ s'annulent tous aux points $H_{1}, \cdots, H_{n+k+1}$ de $C_{n}$. Comme un seul mineur définit au plus $n+k$ points sur $C_{n}$ on en déduit que $M$ n'est pas de rang maximal i.e. que l'application possède un noyau qui par fonctorialité est $\bar{q}_{*} \bar{p}^{*} S^{\vee}$. Comme (d'après la proposition 2.1) $h^{0}\left(S_{H}^{\vee}\right)=1$ pour tout hyperplan $H \in C_{n}$, on a $\bar{q}_{*} \bar{p}^{*} S^{\vee}=O_{C_{n}}\left(-\frac{m}{n}\right)$ pour un entier $m \geq 0$ que l'on va déterminer.

L'image de $M$ est un fibré sur $C_{n}$ de rang $n+k-1$ décomposé défini par $n+k-1$ entiers $a_{1}, \cdots, a_{n+k-1}$ tels que $0 \leq a_{i} \leq 1$ et $m=\sum a_{i}$. On en déduit que $m \leq n+k-1$.

Comme l'unique section $s_{H} \in H^{0}\left(S_{H}^{\vee}\right)$ est partout non nulle (proposition 2.1) la suite exacte canonique sur $X$

$$
0 \rightarrow \bar{q}^{*} O_{C_{n}}\left(-\frac{m}{n}\right) \longrightarrow \bar{p}^{*} S^{\vee} \longrightarrow \mathcal{T} \rightarrow 0
$$

est localement scindée, i.e. le faisceau $\mathcal{T}$ est localement libre sur $X$. On en déduit que l'homomorphisme dual

$$
\bar{p}^{*} S \longrightarrow \bar{q}^{*} O_{C_{n}}\left(\frac{m}{n}\right)
$$

est surjectif. Il induit un homorphisme non nul sur $\mathbf{P}_{n}$

$$
S \longrightarrow E_{m}\left(C_{n}\right)
$$

Remarquons que $m \geq n$. Sinon $E_{m}\left(C_{n}\right)=(m+1) O_{\mathbf{P}_{n}} \oplus(n-m-1) O_{\mathbf{P}_{n}}(-1)([\mathrm{DK}]$, proposition 2.10) donc $h^{0}\left(S^{\vee}\right) \neq 0$ ce qui est faux. Par conséquent $E_{m}\left(C_{n}\right)$ est stable (car pour $m \geq n$ c'est un fibré de Steiner).

Comme $c_{1}(S) \geq c_{1}\left(E_{m}\right)$, c'est une conséquence immédiate de la stabilité des fibrés $S$ et $E_{m}\left(C_{n}\right)$ que l'homomorphisme ci-dessus est un isomorphisme. C'est à dire $m=n+k-1$ et $S \simeq E_{n+k-1}\left(C_{n}\right)$.

Enfin, nous rappelons, sans le démontrer, le résultat suivant du à Dolgachev et Kapranov.

Proposition 2.4. ([DK], prop. 6.8) Tout fibré de Steiner $S \in \mathcal{S}_{n, 2}$ est un fibré de Schwarzenberger $E_{n+1}\left(C_{n}\right)$ pour une c.r.n $C_{n} \subset \mathbf{P}_{n}^{\vee}$.

Contrairement au cas du fibré tangent $\Omega_{\mathbf{P}_{n}}^{\vee}(-1) \simeq E_{n}\left(C_{n}\right)$, tous les hyperplans ne sont pas instables pour $E_{n+1}\left(C_{n}\right)$. En effet on a, d'après la proposition $2.2, W\left(E_{n+1}\left(C_{n}\right)\right)=C_{n}$.

\section{Le résultat central}

Théorème 3.1. Soient $S \in \mathcal{S}_{n, k}$ et $H_{1}, \cdots, H_{n+k+2}$ des hyperplans distincts tels que $h^{0}\left(S_{H_{i}}^{\vee}\right) \neq 0$. Alors il existe une c.r.n. $C_{n} \subset \mathbf{P}_{n}^{\vee}$ telle que $H_{i} \in C_{n}$ pour $i=1, \ldots, n+k+2$ et $S \simeq E_{n+k-1}\left(C_{n}\right)$.

Corollaire 3.1. Soient $\mathcal{H}$ et $\mathcal{K}$ deux arrangements de $m \geq n+2$ hyperplans en position linéaire générale dans $\mathbf{P}_{n}$. Supposons que $E(\mathcal{H}) \simeq E(\mathcal{K})$. Alors une des deux possibilités se produit.

1) $\mathcal{H}=\mathcal{K}$

2) Il existe une c.r.n. $C_{n} \subset \mathbf{P}_{n}^{\vee}$ telle que $E(\mathcal{H}) \simeq E(\mathcal{K}) \simeq E_{m-2}\left(C_{n}\right)$ 
Preuve du corollaire 3.1. Soit $\mathcal{H}=\left\{H_{1}, \cdots, H_{m}\right\}$ un arrangement de $m$ hyperplans en position linéaire générale dans $\mathbf{P}_{n}$ et $E(\mathcal{H})$ le fibré logarithmique associé; rappelons qu'il existe une suite exacte ([DK], prop.2.3)

$$
0 \rightarrow \Omega_{\mathbf{P}_{n}} \longrightarrow E(\mathcal{H}) \longrightarrow \oplus_{i=1}^{m} O_{H_{i}} \rightarrow 0
$$

Ceci prouve que $H_{i} \in W(E(\mathcal{H}))$; si $E(\mathcal{H}) \simeq E(\mathcal{K})$ et $\mathcal{K}=\left\{K_{1}, \cdots, K_{m}\right\}$ on a aussi $K_{i} \in W(E(\mathcal{H}))$ pour $i=1, \cdots, m$.

Supposons que $\mathcal{H} \neq \mathcal{K}$ alors le fibré $E(\mathcal{H})$ possède au moins $m+1$ hyperplans instables distincts. D'après le théorème 3.1 on en déduit qu'il existe une courbe rationnelle normale $C_{n} \subset \mathbf{P}_{n}^{\vee}$ telle que les $m+1$ hyperplans soient des points de $C_{n}$ et $E(\mathcal{H}) \simeq E_{m-2}\left(C_{n}\right)$.

Preuve du théorème 3.1. Comme les fibrés vectoriels de $\mathcal{S}_{n, 1}=\left\{\Omega_{\mathbf{P}_{n}}^{\vee}(-1)\right\}$ et de $\mathcal{S}_{n, 2}$ sont des fibrés de Schwarzenberger on peut supposer $k \geq 3$.

Soient $H_{1}, \cdots, H_{k-2},(k-2)$ hyperplans parmi les $n+k+2$ hyperplans $H_{1}, \cdots, H_{n+k+2}$ instables de $S$. En utilisant 2) de la proposition 2.1 on construit une suite de fibrés de Steiner $S=T_{0}, T_{1}, \cdots, T_{k-2}$

$$
0 \rightarrow T_{i} \longrightarrow T_{i-1} \longrightarrow O_{H_{i}} \rightarrow 0 \quad(i=1, \cdots, k-2)
$$

où $T_{i} \in \mathcal{S}_{n, k-i}$ et en particulier $T_{k-2} \in \mathcal{S}_{n, 2}$. Il existe donc, d'après la proposition 2.4, une c.r.n $C_{n} \subset \mathbf{P}_{n}^{\vee}$ telle que $T_{k-2} \simeq E_{n+1}\left(C_{n}\right)$.

D'après la proposition 2.1 les $(n+4)$ hyperplans restant $H_{k-1}, \cdots, H_{n+k+2}$ sont instables pour $T_{k-2}$. Par conséquent les $(n+4)$ points $H_{k-1}, \cdots, H_{n+k+2}$ sont des points de $C_{n}$. Il faut maintenant vérifier que $H_{1}, \cdots, H_{k-2} \in C_{n}$. Pour ce faire on remarque que parmi $(n+4)$ points de $C_{n}$ tout choix de $(n+3)$ points détermine $C_{n}$. Fixons $H_{k}, \cdots, H_{n+k+2}$. En permutant $H_{k-1}$ avec chacun des points $H_{i}$ pour $i=1, \cdots, k-2$ on prouve que les $n+k+2$ points sont sur la courbe $C_{n}$.

La proposition 2.3 permet de conclure.

\section{References}

[BS] G.Bohnhorst, H.Spindler, The stability of certain vector bundles on $\mathbf{P}_{n}$, in Lecture Notes in Math., 1507, p.39-50, Springer-Verlag, 1992.

[De] P.Deligne, Théorie de Hodge II, Publ.Math. IHES, 40 (1971), 5-58.

[DK] I.Dolgachev et M.Kapranov, Arrangements of hyperplanes and vector bundles on $\mathbf{P}_{n}$, Duke Math.J. 71, (1993), 633-664..

[Sch1] R.L.E.Schwarzenberger, Vector bundles on the projective plane, London Math. Soc. 11, (1961), 623-640.

[Sch2] R.L.E.Schwarzenberger, The secant bundle of a projective variety, Proc. London Math. Soc. 14 (1964), 369-384. 